\title{
Pathogenicity of FtsK mutant of avian pathogenic Escherichia coli
}

\author{
Xiaojing $\mathrm{Xu}^{1}$, Xiang $\mathrm{Chen}^{2}$, Song $\mathrm{Gao}^{2}$, Lixiang Zhao ${ }^{1}$ \\ ${ }^{1}$ College of Basic Medicine and Biological Sciences, Medical Department, \\ Soochow University, 215123, Suzhou, People's Republic of China \\ ${ }^{2}$ Laboratory of Animal Infectious Diseases, College of Veterinary Medicine, \\ Yangzhou University, Yangzhou, 225009, People's Republic of China \\ zhaolixiang@suda.edu.cn
}

Received: October 1, $2015 \quad$ Accepted: February 23, 2016

\begin{abstract}
Introduction: Avian pathogenic Escherichia coli (APEC) is a leading cause of extraintestinal infection and heavy economic losses. Imparting immunity after vaccination with live attenuated strain vaccination is an ideal strategy for infection control. This study considers an FtsK knockout mutant strain as a candidate. Material and Methods: An FtsK knockout mutant of APEC strain E058 was constructed and the pathogenicity of the mutant and wild-type strains was further evaluated in chickens. Results: The $50 \%$ lethal doses of each strain for one-day-old specific-pathogen-free (SPF) chickens challenged experimentally via trachea were $10^{5.5}$ and $10^{7.0}$ colony-forming units (CFU) respectively. Chickens challenged with the wild-type strain exhibited typical signs and lesions of avian colibacillosis, while those inoculated with the mutant strain showed mild pericarditis and pulmonary congestion. The growth rate of the FtsK mutant strain was much slower than the wild-type strain in the heart, spleen, liver, and lung of infected chickens. Conclusion: These results indicated that the APEC FtsK mutant can be attenuated for chickens, and that this mutant has the potential for the development of an APEC vaccine.
\end{abstract}

Keywords: chickens, Escherichia coli, FtsK, mutant.

\section{Introduction}

Extraintestinal pathogenic Escherichia coli (ExPEC) strains can infect nearly every organ and anatomical site in humans and animals. Avian pathogenic E. coli (APEC) strains are one of the leading causes of serious extraintestinal infection of poultry, leading to severe economic losses $(11,14)$. In immunocompromised hosts, APEC strains cause a variety of severe infections with a complex syndrome characterised by multiple organ lesions like air sacculitis, pericarditis, peritonitis, salpingitis, osteomyelitis, or yolk sac infection. APEC of serogroup $\mathrm{O} 2$ is one of the dominant strains, and one which has caused serious economic losses in the poultry industry in China. The ideal strategy for the control of APEC infection could be the establishment of immunity in birds through vaccination with live attenuated strains.

E. coli FtsK is a powerful, fast, double-stranded DNA translocase, which can strip proteins from DNA.
FtsK acts in the late stages of chromosome segregation by facilitating sister chromosome unlinking at the division septum (8). To identify the virulence-associated genes of APEC isolates, selective capture of transcribed sequences (SCOTS) was applied to screen the putative virulence genes expressed in APEC E058 strain (serogroup O2) and several genes were selected, including FtsK (3). These resulting data showed that FtsK may be associated with the virulence of APEC. Thus, the knockout mutant of FtsK may attenuate the virulence of APEC, and the mutant may be a potential agent for vaccine development. In the present study, an FtsK knockout APEC mutant was constructed, and its pathogenicity was evaluated.

\section{Material and Methods}

Bacterial growth condition, plasmids, and primers. APEC E058 and the mutant strains were 
cultured at $37^{\circ} \mathrm{C}$ in Luria-Bertani (LB) broth. The mutant strain was selected on LB agar supplemented with kanamycin at $30 \mu \mathrm{g} / \mathrm{mL}$. Plasmids and primers are listed in Table 1.

Chicken challenge model for FtsK expression level in vivo. The chicken challenge model was used as previously described (17). Briefly, 10 one-day-old SPF chickens were infected with $10^{10} \mathrm{CFU}$ of wildtype or mutant strains by the air sac route. The chickens were euthanised $4 \mathrm{~h}$ later and blood was collected into $15 \mathrm{~mL}$ centrifuge tubes containing $1 \mathrm{~mL}$ of $0.5 \%$ sodium citrate. The blood was centrifuged $\left(5 \mathrm{~min}, 500 \times \mathrm{g}, 4^{\circ} \mathrm{C}\right)$ and the bacteria-containing supernatant was transferred into other tubes. Then, the supernatants were centrifuged again for $10 \mathrm{~min}$ $\left(2500 \times \mathrm{g}, 4^{\circ} \mathrm{C}\right)$, and the final bacterial pellet was stored frozen at $-70^{\circ} \mathrm{C}$ until RNA extraction.

RNA extraction and cDNA synthesis. RNA was extracted using an RNeasy Mini Kit (QIAGEN, USA) according to the manufacturer's instructions. As for cDNA synthesis, about $20 \mu \mathrm{g}$ of the extracted RNA was mixed with about $750 \mathrm{ng}$ of random hexamers (Invitrogen, part of Thermo Fisher Scientific, USA). Then, SuperScript II reverse transcriptase (1 500 U; Invitrogen) was added, along with First Strand buffer, deoxyribonucleotides, and dithiothreitol at recommended concentrations according to the manufacturer's protocol. The cDNA synthesis was performed according to the following programme: $37^{\circ} \mathrm{C}$ for $60 \mathrm{~min}, 42^{\circ} \mathrm{C}$ for $60 \mathrm{~min}$, and $70^{\circ} \mathrm{C}$ for $10 \mathrm{~min}$. The cDNA was further purified using PCR Clean-up NucleoSpin Extract II kits (MachereyNagel, Germany).

RT-PCR and quantitative real-time PCR (qRT-PCR). Primer sets for PCR amplification of irp (encoding iron receptor protein), lolA (encoding outer-membrane lipoprotein carrier protein), and the first 240 amino acids of $f t s K$ protein were irpF/irpR, lolA/lolAR, and F1F/FfR respectively. The PCR products were resolved on $1 \%$ agarose gels and visualised by ethidium bromide staining.

The qRT-PCR primers of the target gene $f t s K$ and the internal standard gene gapA (glyceraldehyde 3-phosphate dehydrogenase) were qFtsKF/qFtsKR and qgapAF/qgapAF respectively. About $30 \mathrm{ng}$ of cDNA and $200 n \mathrm{M}$ of each primer were mixed with $12.5 \mu \mathrm{L}$ of $2 \times$ SYBR Green I PCR Master Mix (Takara Bio, Japan). The cDNA was then amplified in an ABI Prism model 7300 instrument (Applied Biosystems, part of Thermo Fisher Scientific, USA) with specific primers. The relative expression $(\Delta \mathrm{Ct})$ was calculated by subtraction of the $\mathrm{Ct}$ value between gapA and ftsk.

Cloning of FtsK and construction of mutant E058FtsK. The fragments of fts $K 1$ (nucleotides 14 to 721 of $f t s K$ ) and part of ftsK2 (nucleotides 2410 to 3309 of $f t s k$ ) were amplified by PCR using F1F/F1R and $\mathrm{F} 2 \mathrm{~F} / \mathrm{F} 2 \mathrm{R}$ primer sets respectively, and the two fragments were subcloned into the same pBluescript
SK II(-) (Fermentas, part of Thermo Fisher Scientific, Lithuania) plasmid to form $\mathrm{pS}-\mathrm{fts} K$. The kanamycin resistance $\left(\mathrm{Kan}^{\mathrm{r}}\right)$ obtained by PCR was inserted between ftsK1 and ftsK2 of $\mathrm{pS}-f t s K$ to form $\mathrm{pS}-f t s K-\mathrm{kan}^{\mathrm{r}}$. After verification by sequencing, the disrupted $f t s k$ gene containing the inserted $\operatorname{Kan}^{\mathrm{r}}$ gene in $\mathrm{pS}-f t s K-\operatorname{kan}^{\mathrm{r}}$ (Fig. 1) was amplified by PCR and purified for the electroporation to E058 competent cells as described previously (12). After $24 \mathrm{~h}$ of incubation, the resulting $\mathrm{Kan}^{\mathrm{r}}$ colonies were selected for PCR identification using primers F1F and F2R, and the inactivated ftsk mutant was verified by sequencing.

In vitro and in vivo experiments. The in vitro competition tests were conducted as previously described (10). Overnight cultures of the wild-type and mutant bacteria were mixed together at a ratio of approximately $1: 1$, inoculated into fresh $\mathrm{LB}$, and grown with aeration. At various times, the ratio of $\mathrm{Kan}^{+}$and $\mathrm{Kan}^{-}$colonies was determined and subsequently used to calculate the percentage of each strain. For in vivo competition tests, $0.5 \mathrm{~mL}\left(2 \times 10^{8}\right)$ of the wild-type and mutant strains were administered intratracheally to 10 chickens at a ratio of $1: 1$. Five SPF chickens were euthanised 24 and $48 \mathrm{~h}$ later and the heart, liver, lungs, and spleen of the infected chickens were collected. Recoveries of the wild-type and mutant strains were determined by serial dilutions of the collected organ homogenate on LB medium with or without kanamycin respectively. The output ratio (mutant/wild-type) and input ratio (mutant/wildtype) were determined. The competitive index (CI) of the mutant strains was calculated by dividing the output ratio by the input ratio. Based on the CI, when the mutant was outcompeted up to 10 -fold it was evaluated as slightly attenuated, up to 100 -fold as moderately attenuated, and more than 100 -fold as highly attenuated (9).

LD50 test for wild-type and mutant strains. The virulence of the wild-type and mutant strains were evaluated using the $50 \%$ lethal dose $\left(\mathrm{LD}_{50}\right)$ test using one-day-old SPF chickens as previously described (4). Briefly, the bacteria were grown to $\mathrm{OD}_{600}$ of 0.2 in $\mathrm{LB}$, and then pelleted and resuspended in PBS to six different densities $\left(10^{8}, 10^{7}, 10^{6}, 10^{5}\right.$, $10^{4}$, and $10^{3} \mathrm{CFU}$ per $\mathrm{mL}$ ). For each density, five 1-day-old SPF chickens were inoculated into the trachea with $0.1 \mathrm{~mL}$ of each suspension. The chickens were observed for one week and the $\mathrm{LD}_{50}$ values were calculated as previously described (13).

The animal experimentation in this work adhered to the International Guiding Principles for Biomedical Research Involving Animals, as issued by the Council for the International Organisations of Medical Sciences.

Statistical analysis. Statistical significance was evaluated using Student's $t$ test. Differences were considered significant for $\mathrm{P}<0.05$. 


\section{Results}

FtsK upregulated during the infection in chickens. The qRT-PCR was used to detect the relative expression of ftsK of E058 both in vivo and in vitro. It was demonstrated that $f t s K$ was upregulated $23.57 \pm 0.83$ folds during the infection in chickens in vivo, compared with its expression cultured in LB in vitro (Fig. 2).

Construction and characterisation of FtsK mutant. The FtsK mutant was constructed by allelic exchange of a $1635 \mathrm{bp}$ deletion within the fts $K$ coding region with a $\mathrm{Kan}^{\mathrm{r}}$ cassette (Fig. 1). DNA sequencing also confirmed that the cassette was successfully inserted into the right position of mutant strain E058FtsK. RT-PCR showed that E058FtsK could only express the N-terminal 200-amino-acid domain of FtsK, and that transcription of irp and lolA were not disrupted (data not shown).

Attenuated virulence of E058FtsK. The growth capacity of E058FtsK to compete with E058 was compared both in vivo and in vitro. The CIs of mutant in the heart and lungs were $0.08 \pm 0.015$ and $0.09 \pm 0.011$ respectively, indicating that the mutant was moderately attenuated in these tissues. Meanwhile, CIs were 0.0051 \pm 0.0012 and $0.0035 \pm 0.0023$ in the liver and spleen respectively, indicating that E058FtsK was strongly out competed by the wild type (Table 2). In contrast, the in vitro $\mathrm{CI}$ was about 0.30 at $4 \mathrm{~h}$ post inoculation. Although the growth rate of the wild strain was higher than the mutants 10-12 h post inoculation, the difference was not significant (Fig. 3).
To imitate the natural infection, E058FtsK and E058 were administered into the trachea of the chickens. The colonising capacities to internal organs of the wild-type and mutant strains were determined by calculating colony numbers in different tissues 12 and $24 \mathrm{~h}$ after infection (Fig. 4). Both strains can colonise the heart, liver, lungs, and spleen. Significantly, in E058 after $12 \mathrm{~h}$ maximum colonisation of the heart was observed, while E058FtsK exhibited maximum colonisation of the lungs. Minimal colonisation was in the liver and was $6.60 \pm 0.23 \log _{10} \mathrm{CFU} / \mathrm{g}$ for E058 and $4.09 \pm 0.07 \log _{10} \mathrm{CFU} / \mathrm{g}$ for E058FtsK. After $24 \mathrm{~h}$, E058 had colonised the lungs maximally, and E058FtsK had colonised the spleen maximally. Colonisation was much lower at $24 \mathrm{~h}$ than at $12 \mathrm{~h}$ post infection in the heart and lungs for wild-type strains. However, on average the FtsK mutant strain was recovered at 10-1000 times lower levels than that of the wild-type strain from all selected organs at 12 and $24 \mathrm{~h}$ post infection (Fig. 4).

E058FtsK and E058 were further evaluated in vivo by an $\mathrm{LD}_{50}$ test. The $\mathrm{LD}_{50}$ of E058FtsK was $10^{7.0} \mathrm{CFU}$ and the $\mathrm{LD}_{50}$ of E058 was $10^{5.5} \mathrm{CFU}$ in one-day-old SPF chickens. Chickens challenged with wild-type strain exhibited signs characteristic for avian colibacillosis, such as inflammation of the air sacs, haemorrhages, and typical fibrinous lesions. In contrast, mutant E058FtsK only induced mild air sacculitis, pericarditis, pulmonary congestion, and slight hypertrophy of the spleen.

Table 1. Plasmids and primers

\begin{tabular}{|c|c|c|}
\hline & Description & Source \\
\hline \multicolumn{3}{|l|}{ Plasmids } \\
\hline pGEM-T ${ }^{\mathrm{R}}$ EasyVector & TA cloning Vector, $\mathrm{Ap}^{\mathrm{r}}$ & Promega \\
\hline pT-fts $K$ & $f t s K$ cloned into pGEM-T ${ }^{\mathrm{R}}$ Easy Vector & Present study \\
\hline pBluescript II SK(-) & Cloning Vector & Fermentas \\
\hline pS-ftsK & fts $K$ fragment cloned into SK(-) & Present study \\
\hline $\mathrm{pS}-f t s K-\mathrm{Kan}^{\mathrm{r}}$ & Kanamycin resistant gene inserted into $\mathrm{pS}-f t s K$ & Present study \\
\hline \multicolumn{3}{|l|}{ Primers } \\
\hline qFtsKf & ATGATGCGTCCGATTCCAG & Present study \\
\hline qFtsKr & ATTCGTCCACCAACACCAC & Present study \\
\hline qgapAf & CATCGTTTCCAACGCATCCT & Zhao et al. (17) \\
\hline qgapAr & ACCTTCGATGATGCCGAAGTT & Zhao et al. (17) \\
\hline F1F & TAAGCTTTAACCCTTCGGACC & Present study \\
\hline F1R & GAATTCCTCTTCTTCATCATCСАТС & Present study \\
\hline $\mathrm{F} 2 \mathrm{~F}$ & GGATCCCTGTTGATGCGTAATG & Present study \\
\hline F2R & TTCTAGATTCGTCCACCAACACC & Present study \\
\hline $\mathrm{KanF}$ & AATCCGGATCCGTCGACCT & Present study \\
\hline KanR & AGAATTCCGTCGACCTGCAG & Present study \\
\hline irpF & CAAGGGTTTATTCAGGGCTAT & Present study \\
\hline $\operatorname{irpR}$ & CCAGACGGCAAACAGGAC & Present study \\
\hline lolAF & CCGATTGGGAGTGACGT & Present study \\
\hline lolAR & CTAAACTGATGGATGGATTGTGCC & Present study \\
\hline FtsKF & ATGGCTGCCTTACTAAGCTT & Present study \\
\hline FtsKR & TTAGTCAAATGGCGGTGGG & Present study \\
\hline FfR & ACAACGCAGCGTCTGTTTGC & Present study \\
\hline
\end{tabular}


Table 2. Competition assay between E058FtsK and E058 was determined using competitive index (CI)

\begin{tabular}{|c|c|c|c|c|c|c|}
\hline \multirow[t]{2}{*}{ Strains } & \multirow[t]{2}{*}{ LD50 } & \multicolumn{5}{|l|}{ CIs } \\
\hline & & in vitro & heart & lung & liver & spleen \\
\hline WT & $<10^{5.5}$ & - & - & - & - & - \\
\hline FtsK mutant & $>10^{7.0}$ & - & - & - & - & - \\
\hline FtsK mutant/wt & - & $0.30 \pm 0.05$ & $0.08 \pm 0.015$ & $0.09 \pm 0.011$ & $0.0051 \pm 0.0012$ & $0.0035 \pm 0.0023$ \\
\hline
\end{tabular}

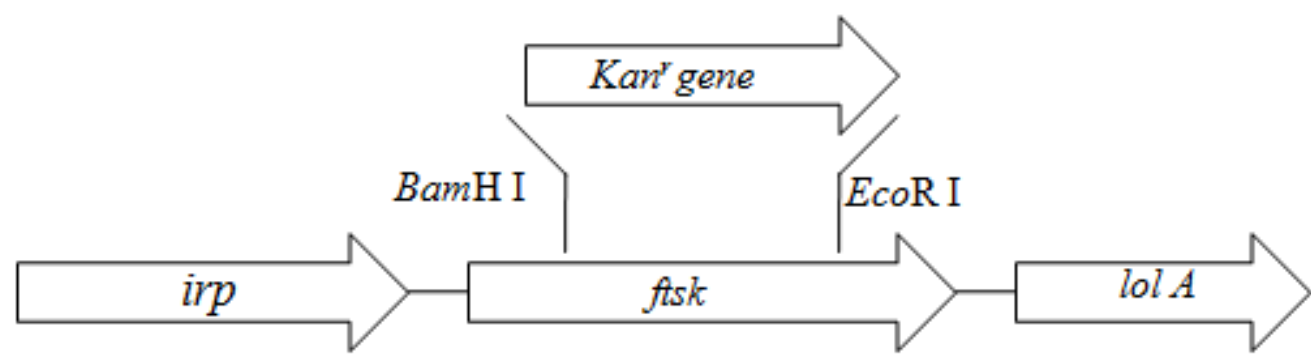

Fig. 1. Genetic organisation of the construction of ftsk mutant. The location of the deletion in ftsk replaced by a Kan gene is between BamH I and EcoR I cleavage sites introduced by PCR. The genes up- and downstream from ftsk are irp and lolA respectively

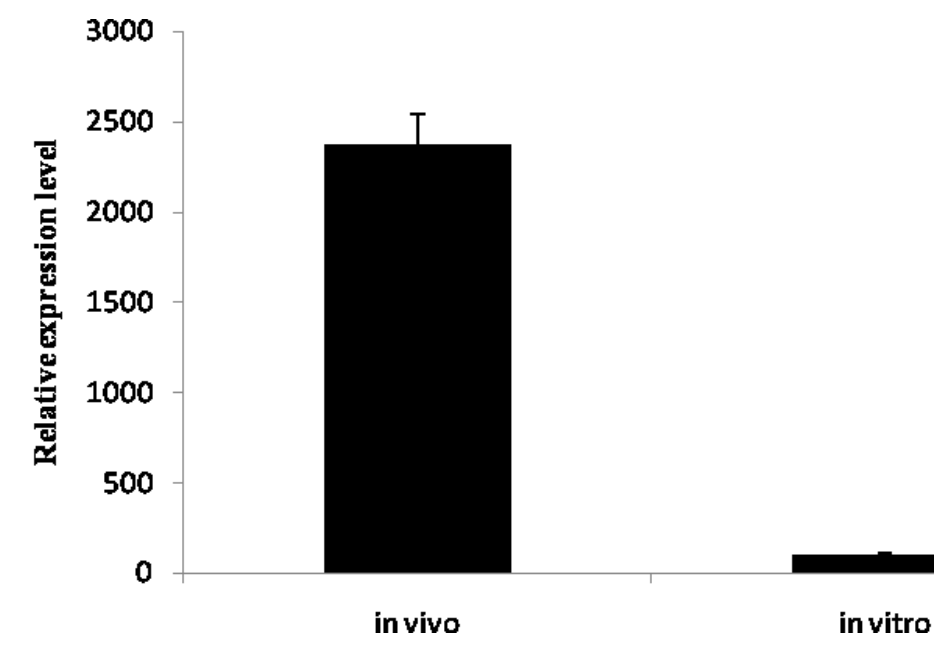

Fig. 2. Quantification of mRNA expression levels of ftsk. Relative expression levels of $f$ tsk were normalised to internal control (gapA)

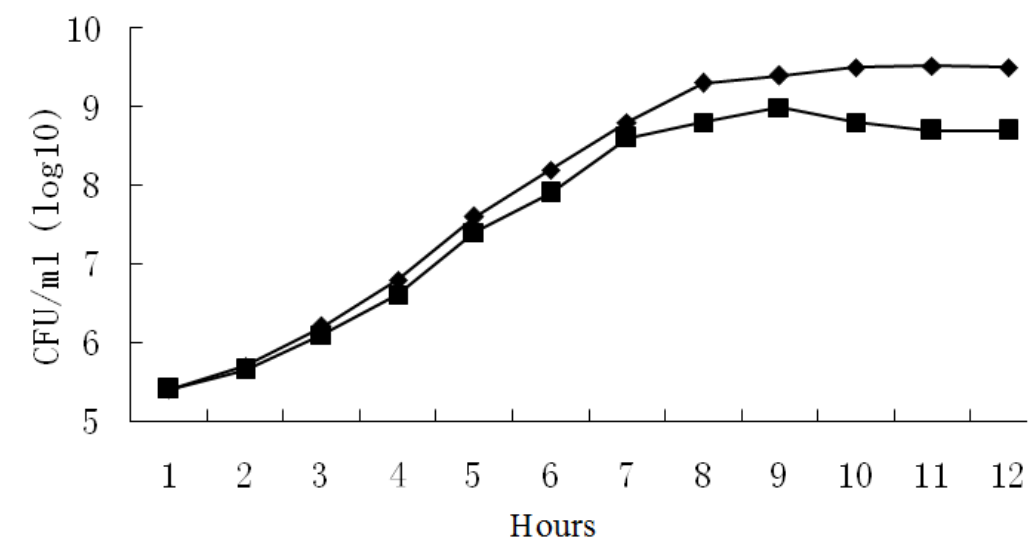

Fig. 3. Growth competition curves of APEC wild-type strain E058 $(\bullet)$ and E058FtsK (घ) in $\mathrm{LB}$ broth at $37^{\circ} \mathrm{C}$ 


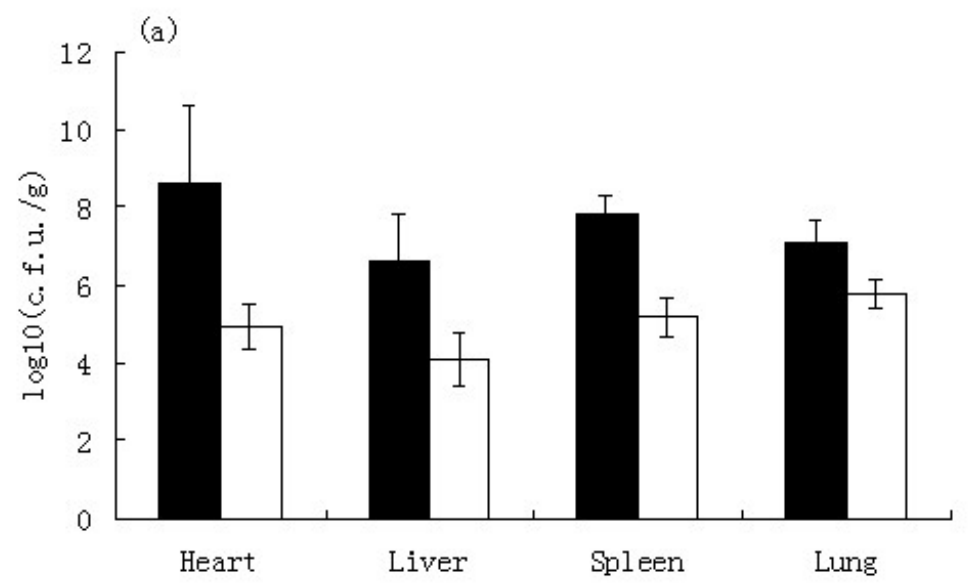

(b)

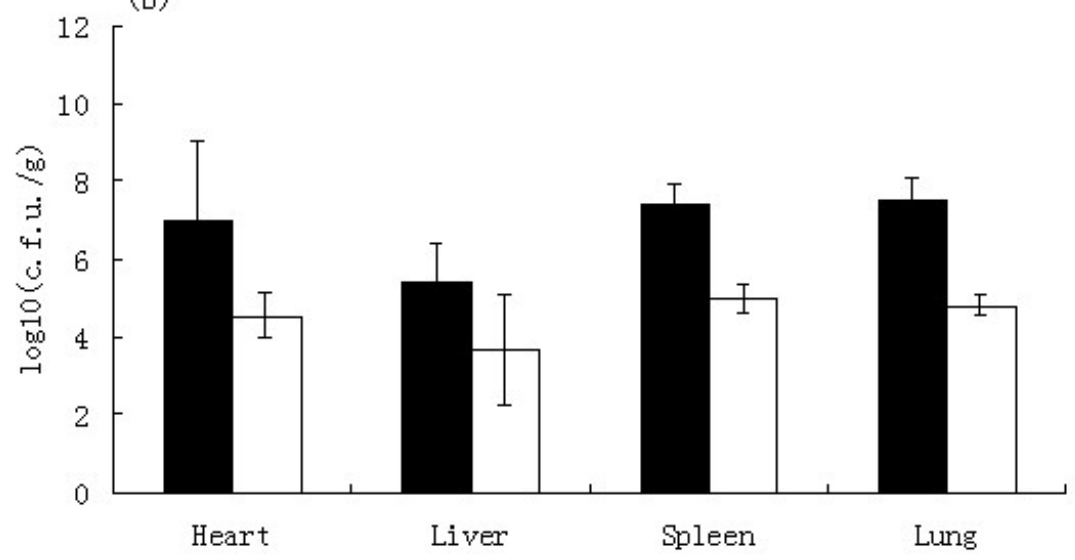

Fig. 4. Bacterial loads in selected tissues from five-week-old chickens $12 \mathrm{~h}$ (a) and $24 \mathrm{~h}$ (b) after infection with E058 (black bars) and E058FtsK (white bars)

\section{Discussion}

Up to now, the vast majority of successful antibacterial vaccines have been attenuated live vaccines based on the principle that attenuated vaccine strains can confer more effective protection against virulent strains. An ideal way to control APEC infection in birds is vaccination with attenuated strains of dominant serogroups. Therefore, determination of the bacterial genes that are essential for APEC pathogenesis may provide valuable information for the prevention and control of infectious diseases caused by APEC. However, the mechanisms underlying the pathogenicity of APEC are still not fully understood, and lots of virulence-associated genes are also awaiting elucidation. In recent years, new molecular approaches have been applied in the identification of genes involved in APEC pathogenesis, including in vivo expression technology, SCOTS, and suppression subtractive hybridisation. Many of these genes have been found, such as genes involved in DNA metabolism and amino acid biosynthesis $(5,16)$, as well as genes involved in iron regulation and stationaryphase growth (7). These genes are often upregulated during APEC infection (15). Previously, we applied
SCOTS to identify putative virulence-associated genes in APEC strain E058 that are expressed in infected chicken tissues, and the fts $K$ gene was selected. In this study, we detected the expression of fts $K$ by qRT-PCR. The results showed that FtsK was upregulated during APEC E058 infection in chickens, compared with its expression in LB, suggesting that FtsK may be involved in the pathogenesis of APEC. However, little is known about the pathogenetic roles of FtsK in APEC. In the present study, an FtsK mutant APEC E058 strain (E058FtsK) was constructed and the virulence of this mutant was analysed.

The in vitro competition results indicate that E058FtsK demonstrates a marginally-decreased growth rate compared with the wild strain in LB. This growth rate suggested that the disruption of ftsk has a minor impact on bacterial growth in vitro. However, in vivo virulence is always not as same as in vitro assays. So, we tested whether the FtsK mutant strain exhibits decreased virulence as compared with the wild-type strain in the chicken model. Chickens were infected intratracheally with either wild-type or FtsK mutant strains. At specific time points post infection, the number of CFU in selected organs showed that the E058 FtsK mutant remained much less prevalent 
than the wild-type strain. These results showed a dramatically-decreased virulence in vivo compared to that of the FtsK mutant in vitro, suggesting that the deletion of the N-terminal of FtsK is more vital to the proliferation of APEC in vivo than in vitro. In other words, FtsK may be not only contributing to the cell division, but also to the resistance of the parenteral environment in vivo. Therefore, the proliferation rate of the cells was decreased in vitro after deletion of the $\mathrm{N}$-terminal of FtsK, and the growth was further delayed in vivo as the bacteria grow parenterally.

Following intratracheal administration of the strains, the $\mathrm{LD}_{50}$ assays indicated that the virulence of the FtsK mutant strain is much lower than that of the wild-type strain. In addition, the pathological lesions in birds infected with E058FtsK were also milder than those infected with the parent strain E058. These results suggest that the virulence of the E058FtsK mutant was attenuated compared with its parent strain E058.

E. coli FtsK is a large protein that is essential for cell division and resolution of chromosome dimers by site-specific recombination. The FtsK protein contains three distinct domains, the first of which is a 279-residue $\mathrm{N}$-terminal integral membrane domain with a series of transmembrane $\alpha$-helices, which functions in cytokinesis and localises FtsK to the septum. Next is a proline- and glutamine-rich domain (of about 650 amino acids) which links the $\mathrm{N}$-terminal to the $\mathrm{C}$-terminal DNA translocase domain that acts in the final stages of chromosome unlinking by chromosome dimer resolution and decatenation. The third domain is the C-terminal (of about 469 amino acids) including a nucleotide-binding consensus sequence $(1,2,6)$. In this study, the FtsK mutant strain E058FtsK only expresses the N-terminal 240-amino-acid domain of FtsK, and lacks the glutamine-rich domain and the C-terminal domain. In vitro and in vivo results showed that the E058FtsK could grow in LB and infect chickens. These results support previous experiments demonstrating that only the N-terminal 200-amino-acid domain of FtsK was required for cell division and that deletion of the protein remainder was not lethal to $E$. coli (6). Indeed, the absence of the C-terminal domain of FtsK could be compensated for by the segregation activities of other genes such as MukB. However, the FtsK mutant was attenuated compared with its parent strain both in vitro and in vivo, suggesting that the proline-and glutaminerich domain and the $\mathrm{C}$-terminal domain play an important role in the pathogenesis of APEC infection, and that this mutant strain may be a potential agent for the development of APEC vaccine.

Conflict of Interests Statement: The authors declare that there is no conflict of interests regarding the publication of this article.
Financial Disclosure Statement: The source of funding of this research and article were the authors' own funds.

Animal Rights Statement: The authors declare that the experiments on animals were conducted in accordance with local Ethical Committee laws and regulations as regards care and use of laboratory animals.

\section{References}

1. Begg K.J., Dewar S.J., Donachie W.D.: A new Escherichia coli cell division gene, ftsK. J Bacteriol 1995, 177, 6211-6222.

2. Bigot S., Sivanathan V., Possoz C., Barre F.X., Cornet F.: FtsK, a literate chromosome segregation machine. Mol Microbiol 2007, 64, 1434-1441.

3. Chen X., Gao S., Wang X.Q., Jiao X.A., Liu X.F.: Identification of APEC genes expressed in vivo by selective capture of transcribed sequences. Acta Microbiol Sin 2007, 47, 407-412.

4. Dho M., Lafont J.P.: Adhesive properties and iron uptake ability in Escherichia coli lethal and nonlethal for chicks. Avian Dis 1984, 28, 1016-1025.

5. Dorman C.J., Corcoran C.P.: Bacterial DNA topology and infectious disease. Nucleic Acids Res 2009, 37, 672-678.

6. Draper G.C., McLennan N., Begg K., Masters M., Donachie W.D.: Only the N-terminal domain of FtsK functions in cell division. J Bacteriol 1998, 180, 4621-4627.

7. Emody L., Kerényi M., Nagy G.: Virulence factors of uropathogenic Escherichia coli. Int J Med Microbiol 2003, 2, 29-33.

8. James E.G., Viknesh S., David J.S., Arciszewska L.K.: FtsK translocation on DNA stops at XerCD-dif. Nucleic Acids Res 2010, 38, 72-81.

9. Li G., Laturnus C., Ewers C., Wieler L.H.: Identification of genes required for avian Escherichia coli septicemia by signature tagged mutagenesis. Infect Immun 2005, 73, 2818-2827.

10. Merrell D.S., Tischler A.D., Lee S.H., Camilli A.: Vibrio cholerae requires rpoS for efficient intestinal colonization. Infect Immun 2000, 68, 6691-6696

11. Mokady D., Gophna U., Ron E.Z.: Virulence factors of septicemic Escherichia coli strains. Int J Med Microbiol 2005 , 295, 455-462.

12. Peng D., Hong W., Choudhury B.P., Carlson R.W., Gu X.X.: Moraxella catarrhalis bacterium without endotoxin, a potential vaccine candidate. Infect Immun 2005, 73, 7569-7577.

13. Reed L.J., Muench H.: A simple method of estimating fifty per cent end points. Am J Hyg 1938, 27, 493-497.

14. Russo T.A., Johnson J.R.: Proposal for a new inclusive designation for extraintestinal pathogenic isolates of Escherichia coli: ExPEC. J Infect Dis 2000, 181, 1753-1754.

15. Tyler D., Lichti C.F., Diekman A.B., Foley S.L.: Evaluation of differentially expressed proteins following serum exposure in avian pathogenic Escherichia coli. Avian Dis 2008, 52, 23-27.

16. Vendeville A., Winzer K., Heurlier K., Tang C.M., Hardie K.R.: Making 'sense' of metabolism: autoinducer-2, LuxS and pathogenic bacteria. Nat Rev Microbiol 2005, 3, 383-396.

17. Zhao L., Gao S., Huan H., Xu X., Zhu X., Yang W., Gao Q., Liu X.: Comparison of virulence factors and expression of specific genes between uropathogenic Escherichia coli and avian pathogenic Escherichia coli in murine urinary tract infection model and chicken challenge model. Microbiology 2009, 155, 1634-1644. 